\title{
Integrated Proteomic and Cytological Study of Rice Endosperms at the Storage Phase
}

\author{
Sheng Bao Xu, ${ }^{\dagger}$ Hua Tao Yu, ${ }^{\dagger, \neq}$ Long Feng Yan, ${ }^{\dagger, \neq}$ and Tai Wang*, \\ Research Center for Molecular \& Developmental Biology, Key Laboratory of Photosynthesis \& Environmental \\ Molecular Physiology, Institute of Botany, Chinese Academy of Sciences and National Center for Plant Gene \\ Research, Beijing 100093, China, and Graduate School of Chinese Academy of Sciences, Beijing 10049, China
}

Received December 6, 2009

The endosperm at the storage phase undergoes a series of coordinated cellular and metabolic events, including starchy endosperm cell death, starch synthesis, and starch granule packaging, which leads to efficient accumulation of starch. However, the mechanism underlying the interconnections remains unknown. We used integrated proteomic and cytological approaches to probe the interconnections in rice (Oryza sativa) endosperm at the storage phase from 12 to 18 days after flowering (DAF). Starch granule packaging was completed first in the inner part of endosperm at $15 \mathrm{DAF}$ and spread to almost the entire endosperm at 18 DAF. Programmed starchy endosperm cell death occurred after the starch granule packaging. Endogenous $\mathrm{H}_{2} \mathrm{O}_{2}$ was detectable in the inner part of endosperm at $12 \mathrm{DAF}$ and the region beyond the inner part at $15 \mathrm{DAF}$, with an $\mathrm{H}_{2} \mathrm{O}_{2}$ burst at $15 \mathrm{DAF}$. Proteomics analysis with 2-D fluorescent difference gel electrophoresis and matrix-assisted laser-desorption ionization time-of-flight/ time-of-flight mass spectrometry revealed 317 proteins, including almost all known antioxidants, differentially expressed throughout the 3 stages of the developmental phase. More than two-thirds of the 317 proteins were potential thioredoxin targets, with a preferential skew toward central carbon metabolism, alcoholic fermentation, starch metabolism, amino acid metabolism, and protein synthesis or folding. These proteins implicated in starch synthesis and gluconeogenesis were upregulated, whereas those involved in anabolism of biomacromolecules such as proteins, lipids, and cell wall components were downregulated, with upregulated expression of proteins involved in catabolism of these biomacromolecules, which suggests remobilization of nutrients for starch synthesis. These data suggested important roles of the $\mathrm{H}_{2} \mathrm{O}_{2}$-antioxidant interface in coordinating starch accumulation, programmed cell death of starchy endosperm, and remobilization of nutrients during the cell death.

Keywords: Proteomics $\bullet \mathrm{H}_{2} \mathrm{O}_{2} \bullet$ endosperm $\bullet$ programmed cell death $\bullet$ starch granule $\bullet$ Oryza sativa

\section{Introduction}

The endosperm is initiated by the fusion between a sperm nucleus and 2 polar nuclei during the double fertilization process. ${ }^{1}$ In dicot species, the endosperm is consumed in part or in full by the developing embryo during seed maturation, whereas in monocots the endosperm is a persistent seed structure and represents the main part of the mature seed. The endosperm is important in supporting postembryonic growth and development before seedlings start photosynthesis. ${ }^{2}$

Cereal endosperms belong to the nuclear type of endosperms. After fertilization, the endosperm undergoes a series of cellular or biochemical processes, including syncytium formation, cellularization, and the accumulation of storage reserves, and finally becomes mature. ${ }^{1-3}$ Cell division and the storage phases of endosperm development are key points in

* To whom correspondence should be addressed. E-mail: twang@ ibcas.ac.cn. Tel: 0086-10-62836210. Fax: +86-10-62594170.

${ }^{\dagger}$ Chinese Academy of Sciences and National Center for Plant Gene Research.

${ }^{\ddagger}$ Graduate School of Chinese Academy of Sciences. determining seed weight. In the storage phase, starch is synthesized from imported sucrose by a group of enzymes and then trimmed and packed into semicrystalline starch granules in amyloplasts, which allows for dense packaging of glucose units. ${ }^{4}$ Most endosperm cells are fated to die at the completion of starch and protein accumulation, ${ }^{1}$ and the cell death process of starchy endosperm shares typical features of programmed cell death (PCD). ${ }^{5}$

The mature cereal endosperm consists of 2 main distinct cell types: nonliving starchy endosperm cells and living cells of the aleurone layer at the endosperm periphery. Why starchy endosperm cells die with the accumulation of storage starch is an intriguing question. In general, the default cell death may be required for access of the aleurone/scutellum-generated hydrolases into the starchy endosperm to rapid mobilize the storage reserves necessary to support postembryonic growth and development during germination. ${ }^{3,5}$ However, premature induction of starchy endosperm cell death jeopardizes germination. ${ }^{5}$ In maize endosperm, the pattern and progression of PCD follows that of cellular development and deposition of storage reserves, and PCD is initiated first with the central 
starchy endosperm cells that first finish accumulating starch. ${ }^{6}$ This finding suggests spatial and temporal coordination of starchy endosperm PCD and starch synthesis, but the importance of the coordination is largely unknown.

Reactive oxygen species (ROS) are important signal molecules initiating PCD. ${ }^{7,8}$ ROS-antioxidant interactions act as a metabolic interface that functions in diverse metabolic or cellular processes beyond PCD. ${ }^{9-11}$ However, evidence of the involvement of ROS in starchy endosperm PCD is lacking. ${ }^{5}$ Several observations revealed that the development of cereal seeds undergoes a classic oxidative process, which is required for the regulation of storage protein stability. ${ }^{12,13}$ Redox homeostasis has been proposed as the preferred strategy of plastidial enzymes to regulate diverse metabolisms such as carbon storage and partitioning in leaves and potato tubers. ${ }^{14}$ In senescent leaves, ROS play critical roles in regulating nutrition remobilization. ${ }^{15}$ The roles of ROS or the ROSantioxidant interaction in starchy endosperm PCD and carbon partitioning into starch synthesis in cereal endosperm remain unknown.

Rice is an excellent model for studying endosperm development because of its importance as a staple food and because of the available genome sequence database. Recent studies showed that the rice endosperm reaches a final size in the sink at about 12 days after flowering (DAF) and thereafter is in storage and desiccation phases. ${ }^{16}$ Similar to maize and wheat endosperm cells, rice starchy endosperm cells display similar PCD features such as chromatin condensation, nuclear envelope disruption, and nuclear matrix leakage at the late stage of filling. ${ }^{17}$ We aimed to understand the molecular mechanism of the interconnections of different cellular or biochemical events associated with starch synthesis, starchy endosperm cell death, and nutrition remobilization during the death. We analyzed (1) dynamic proteomic changes by use of fluorescent 2-D difference gel electrophoresis (2D-DIGE), (2) spatial and temporal dynamics of starch granule packaging, and (3) $\mathrm{H}_{2} \mathrm{O}_{2}$ changes in endosperm at the storage phase from 12 to $18 \mathrm{DAF}$.

\section{Materials and Methods}

Plant Materials and Sampling. The rice cultivar Zhonghua 10 (Oryza sativa L. ssp japonica) was cultured during the rice growing season (May-September) as described previously. ${ }^{16}$ The superior caryopses (also called seeds) of the top 3 spikelets were labeled as described by $\mathrm{Xu}$ et al. ${ }^{16}$ Labeled spikelets were harvested at 12, 15, and $18 \mathrm{DAF}$, respectively. At least 300 caryopses from 50 spikelets were collected at each of the 3 stages and divided into 6 aliquots, which were stored immediately at $-80{ }^{\circ} \mathrm{C}$.

Observation of Endosperm Cell Viability and Characteristics. To characterize endosperms at each stage (12, 15, or 18 DAF), the longitudinal section and transection of each endosperm were photographed under an Olympus SZX9 microscope. To assess the endosperm cell viability, cross sections located at the longitudinal median of the endosperm were stained by Evans blue as described previously. ${ }^{6}$ At least 20 caryopses were observed at each stage.

For scanning electron microscopy (SEM), one-fifth of the dehusked caryopses (the tip) was cut and fixed in a solution of formalin/acetic acid/alcohol (FAA, 5\% formaldehyde, 44.5\% ethanol, and $6 \%$ acetic acid) for 4 days at room temperature (about $25^{\circ} \mathrm{C}$ ). The samples after fixation were dehydrated in a series of graded ethanol from $60 \%$ to $100 \%$ alcohol and incubated in a series of alcohol-isoamyl acetate mixtures, with the volume proportion of $75 \%-25 \%, 50 \%-50 \%, 25 \%-75 \%$, and 0-100\%. Samples were dried with $\mathrm{CO}_{2}$ to a critical point, divided into 2 segments from the middle of a caryopsis, mounted on SEM stubs, and sputter coated with gold (HITACHI E-1010, Japan). The cross sections of these specimens were observed on SEM (HITACHI S-4800, Japan) at an accelerating voltage of $10 \mathrm{kV}$.

$\mathbf{H}_{2} \mathbf{O}_{2}$ and ATP Measurement. To examine $\mathrm{H}_{2} \mathrm{O}_{2}$ distribution in developing endosperm, endosperm was stained with $3^{\prime} 3$ diaminobenzidine (DAB) as described ${ }^{18}$ with some modification. Briefly, after endosperm was cut at the longitudinal median, the semiendosperm was immersed in DAB solution ( $1 \mathrm{mg} / \mathrm{mL}, \mathrm{pH}$ 3.8; Sigma, St. Louis, MO) for $12 \mathrm{~h}$ and then soaked in $100 \%$ ethanol for $4 \mathrm{~h}$ to destain. The cross sections were photographed under an Olympus SZX9 microscope, and at least 20 independent replicates were tested for each sample. $\mathrm{H}_{2} \mathrm{O}_{2}$ production was quantitatively evaluated by use of an Amplex red $\mathrm{H}_{2} \mathrm{O}_{2}$ / peroxidase assay kit (Molecular Probes), with 4 independent replicates performed for each sample.

The ATP content was measured by the luciferase reaction of the ENLITEN ATP assay kit (Promega; http://www. promega.com/) as described. ${ }^{16}$ The bioluminescence of each sample was detected via fluorometer (PolarSTAR, BMG Lab Technologies, http://www.bmglabtech.com). A standard curve was established with use of the ATP standard solution supplied in the kit to quantify the ATP levels. For each sample, triplicate independent biological repeats were performed.

Protein Preparation for Fluorescent 2-D Difference Gel Electrophoresis Analysis (2D-DIGE). Caryopses were dehusked, and endosperm parts were collected by cutting away onethird containing the embryo. The endosperm samples (0.6 g) were ground with ice-cold extraction buffer $(20 \mathrm{mM}$ Tris- $\mathrm{HCl}$, pH 8.0, $20 \mathrm{mM} \mathrm{NaCl}, 10 \mathrm{mM}$ PMSF, 1 mM EDTA) on ice. After centrifuging at $35,000 \mathrm{~g}$ at $4{ }^{\circ} \mathrm{C}$ for $20 \mathrm{~min}$, the supernatant was transferred to a new tube. The pellet was resuspended with the ice-cold extraction buffer, and the supernatant was collected by centrifugation at $35,000 \mathrm{~g}$ at $4{ }^{\circ} \mathrm{C}$ for $20 \mathrm{~min}$. The combined supernatant was supplemented with 4 volumes of ice-cold $10 \% \mathrm{w} / \mathrm{v}$ trichloroacetic acid in $100 \%$ acetone to precipitate proteins at $-20{ }^{\circ} \mathrm{C}$ for $4 \mathrm{~h}$, and proteins were collected by $20 \mathrm{~min}$ of centrifugation at $35,000 \mathrm{~g}$. After two thorough washes with $80 \%$ ice-cold acetone, the resulting proteins were dried at room temperature and dissolved in lysis buffer (7 M urea, $2 \mathrm{M}$ thiourea, 4\% w/v CHAPS, $20 \mathrm{mM}$ Tris$\mathrm{HCl}, \mathrm{pH}$ 8.5), and the debris was removed by centrifugation at $40,000 \mathrm{~g}$ for $20 \mathrm{~min}$. Finally, the $\mathrm{pH}$ of protein samples was adjusted to 8.5 with $\mathrm{HCl}$ and $\mathrm{NaOH}$. The proteins were quantified according to the Bradford method ${ }^{19}$ by DU640 UV-vis spectrophotometry (Beckman) with bovine serum albumin used as a standard. The final proteins underwent 2DDIGE immediately or were stored in aliquots at $-80^{\circ} \mathrm{C}$. For each sample, 4 independent protein preparations were prepared.

2D-DIGE and Image Analysis. To minimize system and inherent biological variation, 4 independent protein preparations for each sample were combined as recommended. ${ }^{20}$ The proteins were labeled with Cy3 and Cy5 minimal fluorescent dye (400 pmol dye $/ 50 \mu \mathrm{g}$ protein) according to the manufacturer's instructions (GE Healthcare). The internal standard was prepared by mixing equal amounts of all analyzed samples and labeling with Cy2 minimal fluorescent dye. Finally, the labeled proteins and the internal standard were supplemented with 2 volumes of sample buffer (8 M urea, $130 \mathrm{mM} \mathrm{DTT,} 4 \% \mathrm{w} / \mathrm{v}$ CHAPS, 2\% Pharmalyte, pH 3-10). For 2D-DIGE analysis, each 
labeled protein sample was mixed with the internal standard (for details, see Supplemental Figure S1), adjusted to a total volume of $450 \mu \mathrm{L}$ with a rehydration buffer (8 M urea, $13 \mathrm{mM}$ DTT, $4 \% \mathrm{w} / \mathrm{v}$ CHAPS, $0.5 \%$ Pharmalyte $\mathrm{pH} 3-10$ ), and then loaded on an IPG strip holder containing a 24-cm pH 4-7 linear gradient IPG strip (GE Healthcare). Isoelectric focusing and SDS-PAGE involved the Ettan IPGphor isoelectric focusing system in an Ettan DALT Six electrophoresis unit as described. ${ }^{16}$

Fluorescence images of gels were acquired on a Typhoon 9400 scanner (GE Healthcare). Cy2, Cy3, and Cy5 images for each gel were scanned at 488/520,532/580, and 633/670 nm excitation/emission wavelengths, respectively, with adjustment of proper voltage to create a maximum signal of about 70,000. Finally, 18 images (derived from 6 gels, each gel contained 2 different samples and one internal standard sample) with similar strength of total signal were obtained (Supplemental Figure S1).

The images were analyzed by use of DeCyder 6.5 software following the DeCyder User Manual (GE Healthcare). A differential gel analysis module was used for intragel codetection of samples and internal standard protein spots, and the estimated number of spots was set as 10,000. Artificial spots (maximum slope $>2.5$, maximum peak height $<150$ ) were removed. Finally, about 2,300 spots were detected in each image. The intergel matching of the internal standard and samples across all gels was analyzed in the biological variation analysis module, and matched spots were further checked manually. Only spots present in all of the 18 gel images were considered. The relative volume (RV), which represents a ratio of signal intensity in the sample and internal standard images of each protein spot in each DIGE gel, was used to quantify the protein expression according to the DeCyder User Manual (GE Healthcare). Following comparative cross-gel statistical analyses of all spots, correction for false discovery rate was applied to eliminate spots that showed significant differential expression that might reach significance by chance. ${ }^{21}$ Spots that showed significant differential expression were determined by ANOVA and Student's $t$ test $(p<0.05)$.

Protein Identification. The gels used for 2D-DIGE analyses were stained with colloidal Coomassie Brilliant Blue for spots with high abundance and with deep purple for spots with low abundance. Then additional gels prepared with $1 \mathrm{mg}$ internal standard proteins were stained deep purple for spots that could not be determined from 2D-DIGE gels.

In-gel digestion was as described previously. ${ }^{22}$ The matrixassisted laser-desorption ionization (MALDI) mass spectra were produced on an Ultroflex II MALDI time-of-flight/time-of-flight mass spectrometer (MALDI-TOF/TOF MS) (Bruker Daltonics, Germany) with use of FlexAnalysis 2.4 software (parameters shown in Supplemental Figure S2). To ensure the accuracy of protein identification, MALDI-TOF/TOF MS was calibrated externally by use of a standard peptide mixture (Bruker); the deviations of all the external calibration peaks were limited in $10 \mathrm{ppm}$. Trypsin autolysis peptides (Roche) and keratins were used for internal calibration, and the maximum deviation was no more than $20 \mathrm{ppm}$. After tryptic peptide masses were transferred to a BioTools 3.0 interface (Bruker Daltonics), peptide mass fingerprintings (PMFs) were searched against the NCBInr protein database (http://www.ncbi.nlm.nih.gov/; NCBInr 20071214; 5,742,110 sequences) by use of Mascot software 2.2.03 (http://www.matrixscience.com; Matrix Science, London, U.K.). Oryza sativa (133,861 sequence entries) was chosen as the taxonomic category. All peptide masses were considered monoisotopic and $[\mathrm{M}+\mathrm{H}]^{+}$(protonated molecularions). Searches involved a mass accuracy of $\pm 100 \mathrm{ppm}$, and one missing cleavage site was allowed for each search. Carbamidomethyl (C) was set as a fixed modification and oxidation (M) and Pyro-glu (N-term Q) as variable modifications. To improve the identification accuracy, the identified proteins had to meet the following standards: (1) probability-based MOWSE score $>65(P>0.05),(2)$ order of magnitude of the following nonhomologous protein less than or not significantly different from $(P>0.05)$ that of the first protein, and (3) most abundant peptides in the spectra assigned as the identified protein (the first hit).

When PMFs were not enough to identify a protein spot under the described criteria, 3 parent peptides with top intensity of the spectrum were selected to further obtain MS/MS fragment ion patterns on Ultraflex MALDI TOF/TOF MS after trypsinand keratin-derived peaks were removed by use of the contaminant database list of PeakErazor (http://www.protein. sdu.dk/gpmaw/Help/PeakErazor/peakerazor.html) with precision of $60 \mathrm{ppm}$. These MS/MS data were analyzed by use of FlexAnalysis 2.4 and searched against the NCBInr database $(O$. sativa, 133,861 sequence entries in NCBI in December 2007) with use of MASCOT 2.2.03 (http://www.matrixscience.com; Matrix Science, London, U.K.). The search parameters in MS/ MS were as for MS searching, with the MS/MS tolerance 0.8 Da (Supplemental Figure S3). Protein scores of more than 44 were considered significant $(P<0.01)$.

Bioinformatics Analysis. To confirm the nonredundant proteins, the chromosome loci of the protein-encoding genes were searched in the TIGR database (http://tigrblast.tigr.org/ euk-blast/index.cgi?project=osal), and loci with the highest scores were accepted as positive results. Different proteins mapped in the same locus were considered unique.

Cluster analysis was performed with the mean of relative volume value of each spot from 4 replicates by use of GeneCluster 2.0 (http://www.broadinstitute.org/cancer/software/ genecluster2/gc2.html), which allows for visualizing the profile of each cluster. After normalization to mean 0 and variance 1 , all clusters except the cluster range $2-5$ were found with default parameters. Different cluster ranges were compared, and a final 4 were selected on the basis of significant difference in functional categories.

Real-Time Quantitative RT-PCR. All procedures were used as described. ${ }^{16}$ An amount of $1 \mu$ g total RNA was used to synthesize the first-strand cDNA with use of SYBR Green Realtime PCR Master Mix (Toyobo). The ACT1 gene was used as a reference. The primer sequences are in Supporting Information.

\section{Results}

Characteristics of Rice Endosperm Cells at 12, 15, and 18 DAF. Superior caryopses (also called seeds) in rice have a high growth rate and reach maturity at the same time. ${ }^{16,23}$ In general, they reach the size of mature seeds at about $12 \mathrm{DAF}$, and thereafter the endosperm enters the storage and desiccation phase. The seed at 18 DAF reaches $>95 \%$ mature seeds in fresh and dry weight. ${ }^{16,23}$ Longitudinal sections and transections of fresh endosperm at 12 DAF appeared evenly milk-white (Figure 1a). A translucent endosperm region (TER), which represents the starch-filled and desiccated endosperm part, covered half of the central endosperm sections at 15 DAF and almost completely overlaid the whole endosperm at 18 DAF 


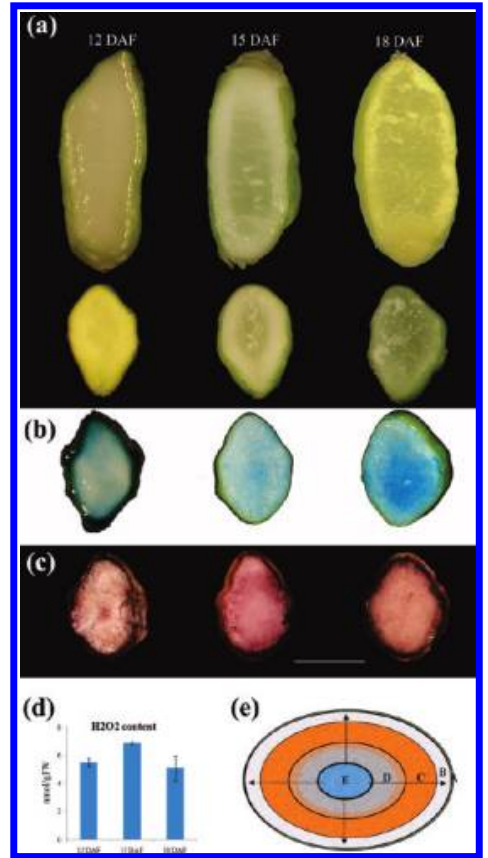

Figure 1. Cellular and biochemical features of rice endosperm the storage phase 12-18 days after flowering (DAF). (a) Longitudinal section and transectional images of endosperm at 12,15 , and $18 \mathrm{DAF}$. (b) Evans-blue staining. (c) DAB staining. (d) Changes in $\mathrm{H}_{2} \mathrm{O}_{2}$ levels. (e) Schematic representation at 15 DAF. A, pericarp and aleurone; $B$, white endosperm; $C$, the region of $\mathrm{H}_{2} \mathrm{O}_{2}$ appearance detected by $D A B$; $D$, translucent endosperm portion (including $\mathrm{E}$ ); $\mathrm{E}$, dead starchy endosperm portion detected by Evans-blue staining. The arrows show the progression of $\mathrm{H}_{2} \mathrm{O}_{2}$ occurrence, translucent endosperm, and starchy endosperm programmed cell death. FW, fresh weight. Data are mean and standard deviation of 4 replicates. The scale bar in panel $\mathrm{c}=2 \mathrm{~mm}$ for panels $\mathrm{a}-\mathrm{c}$.
(Figure 1a). Therefore, no TER, half TER, and nearly full TER are the basic features of 12, 15, and 18 DAF endosperm, respectively.

Evans-blue stain, which stains senescent or dead cells with lost integrity of plasma membranes, ${ }^{6,24}$ showed only a few such cells in endosperm at 12 DAF (Figure 1b). The stained region appeared in the center of the TER at 15 DAF and extended outward from the center with TER enlargement at 18 DAF. The stained region was always smaller than TERs in endosperm at 15-18 DAF (Figure 1b). Thus, only the inner TER cells were senescent or dead, whereas the outer TER cells were still viable at these phases (Figure 1b). Therefore, cell death or senescence in rice endosperm occurs in a regular pattern: first at the inner part and then extending outward after enlargement of the TER.

ROS induction is an important factor triggering cell senescence or death..$^{7,9,25}$ Here, we examined spatiotemporal changes in $\mathrm{H}_{2} \mathrm{O}_{2}$ level in developing endosperm by DAB staining (Figure 1c) and quantitative assay (Figure $1 \mathrm{~d}$ ). DAB staining appeared in the central part of endosperm at $12 \mathrm{DAF}$ and in most endosperm cells that formed a circle band around the central part at $15 \mathrm{DAF}$. At $18 \mathrm{DAF}$, staining appeared only in the outer part of endosperm. The $\mathrm{H}_{2} \mathrm{O}_{2}$ burst occurred at 15 DAF (Figure 1d). This result, in combination with cell viability data seen on Evans-blue staining, showed that the $\mathrm{H}_{2} \mathrm{O}_{2}$ burst occurred just before endosperm cell death or senescence and was concurrent with endosperm cell death.

To evaluate the state of starch deposition and packaging, we observed 3 distinct regions, from the outer (zone A) to middle (zone B) to inner (zone C) parts (Figure 2a, and Figure 3) of median sections of endosperm at 12, 15, and 18 DAF by SEM. In general, the TER in fresh endosperm sections showed a crystallized appearance in the SEM samples (Figure 2a). In 12 DAF-A (refers to zone A of 12 DAF endosperm, same below),

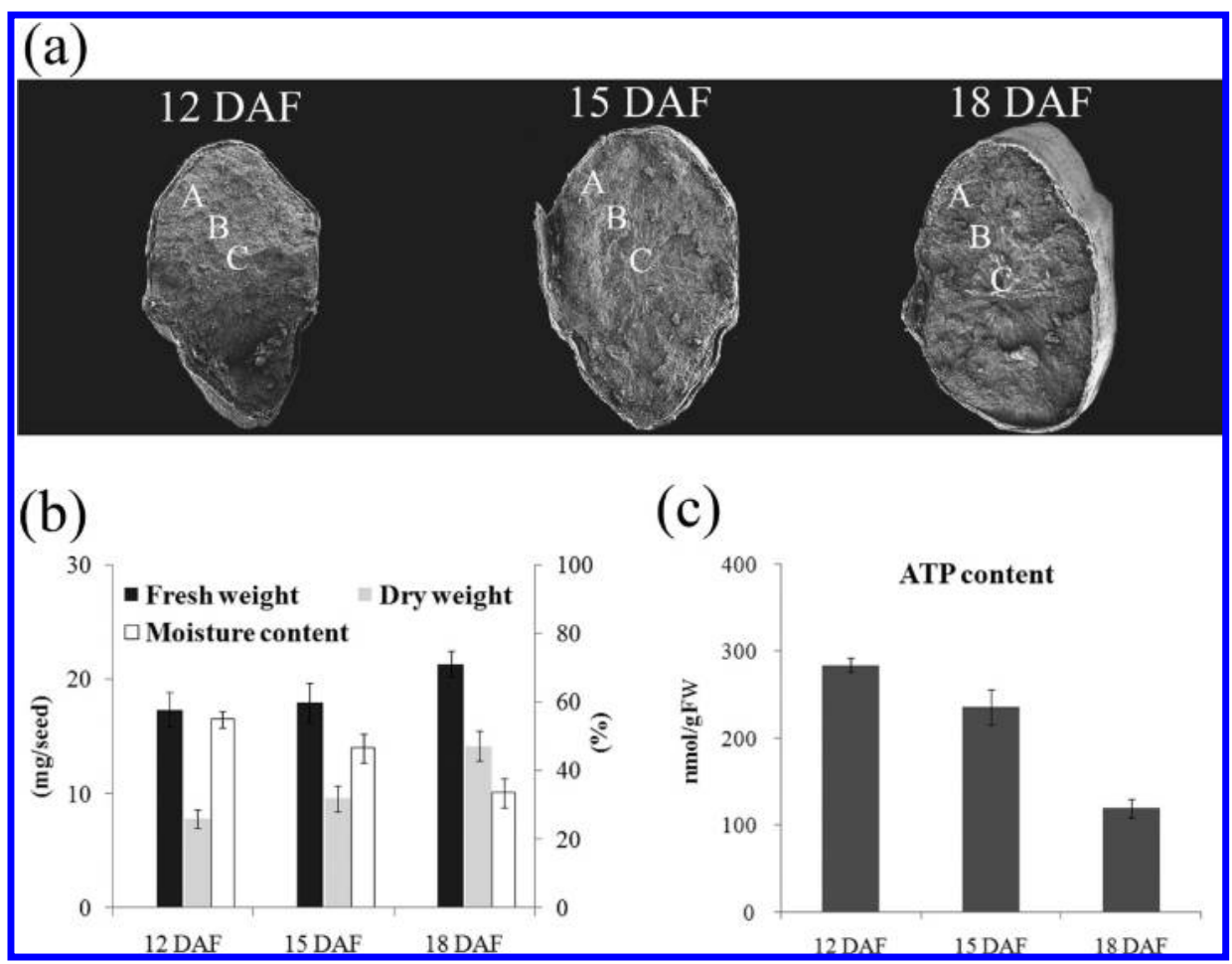

Figure 2. Overall characteristics of endosperm at the storage phase. (a) SEM images of transections of endosperm at 12, 15, and 18 DAF. A, B, and $\mathrm{C}$ represent the outer, middle, and inner parts of endopserm, respectively. (b) Changes in fresh dry weight (left vertical axis, mg/seed) and moisture content (right vertical axis, \%) of developing endosperm. At least 50 seeds were analyzed at each stage. (c) Changes in ATP levels in developing endosperm. FW, fresh weight. Data are mean and standard deviation of 3 independent biological replicates. 


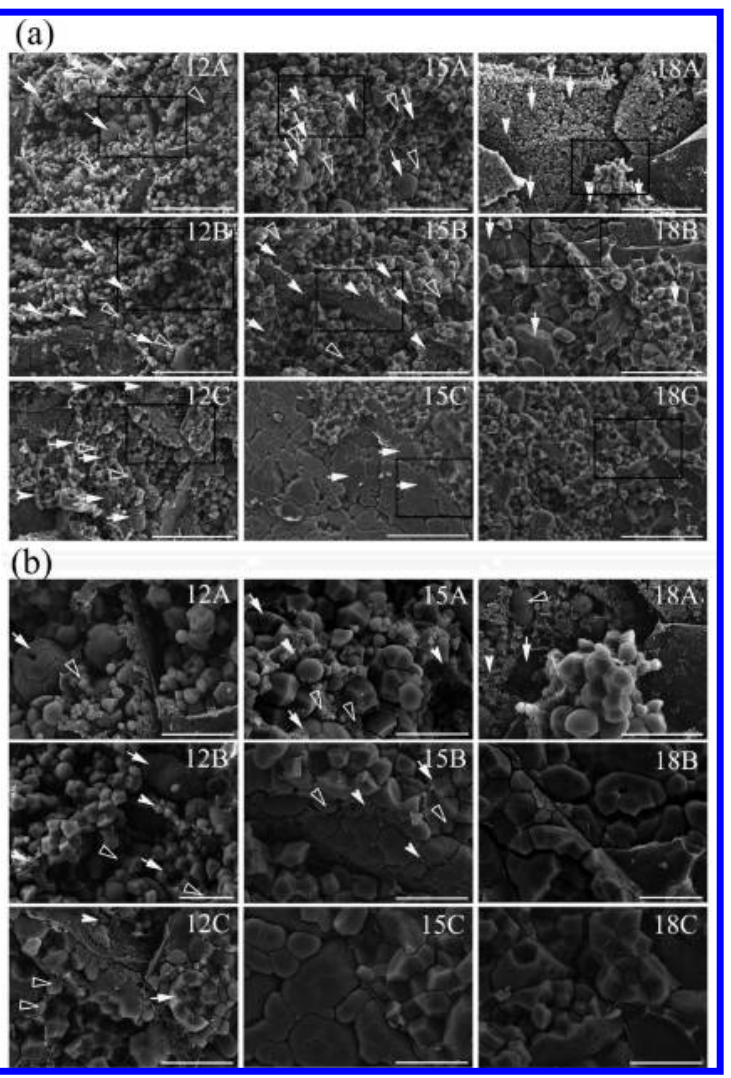

Figure 3. Progression in starch granule packaging observed on SEM. (a) The state of starch granule packaging in cells localized at $A, B$, and $C$ (see Figure $2 a$ ) of endosperm at 12, 15, and 18 DAF. (b) Close-up of the sections marked in panel a (black line rectangle). White arrows showing "football-like" starch granules, white line arrowheads inter-football starch granules, and white arrowheads cortical granules. Scale bar $=30 \mu \mathrm{m}$ in panel a and $10 \mu \mathrm{m}$ in panel $\mathrm{b}$.

most starch granules were packed together and appeared "football-like", with numerous smaller starch granules wrapped by white material around the "football-like" granules (Figure 3b). In 12 DAF-B and 15 DAF-A, the smaller starch granules swelled as the white, wrapped material decreased, and several new starch granules appeared in the cortical endoplasmic reticulum (here termed cortical granules) located near the plasma membrane (Figure 3b), which is consistent with a previous observation of rice endosperm cells. ${ }^{26}$ The results are similar in initiation time and location to a previous observation of the 3 types of granules in wheat endosperm. ${ }^{27}$ Cortical granules were enlarged in 12 DAF-C, 15 DAF-B, and 18 DAF-A (Figure $3 \mathrm{a}$ and $\mathrm{b}$ ). The white materials were almost gone, as tightly packed and enlarged starch granules appeared in 15 DAF-C and 18 DAF-B/C (Figure 3a and b), which is consistent with increased grain weight (Figure 2b). All starch granules in $15 \mathrm{DAF}-\mathrm{C}$ and $18 \mathrm{DAF}-\mathrm{B} / \mathrm{C}$ were similar in size (Figure $3 \mathrm{~b}$ ), which reflects the completion of starch granule packaging. ${ }^{4}$ Therefore, the TER reflected the state of starch deposition and granule packaging, and the completion of starch granule packaging is associated temporally and spatially with endosperm cell senescence or death.

2D-DIGE Analysis Revealed 322 Differentially Expressed Spots. Our previous comparison of $\mathrm{pH} 3-10$ and $\mathrm{pH} 4-7$ stripbased 2-DE separation of rice seed proteins showed that 2-DE with $\mathrm{pH}$ 4-7 strips had better resolution for rice seed pro-

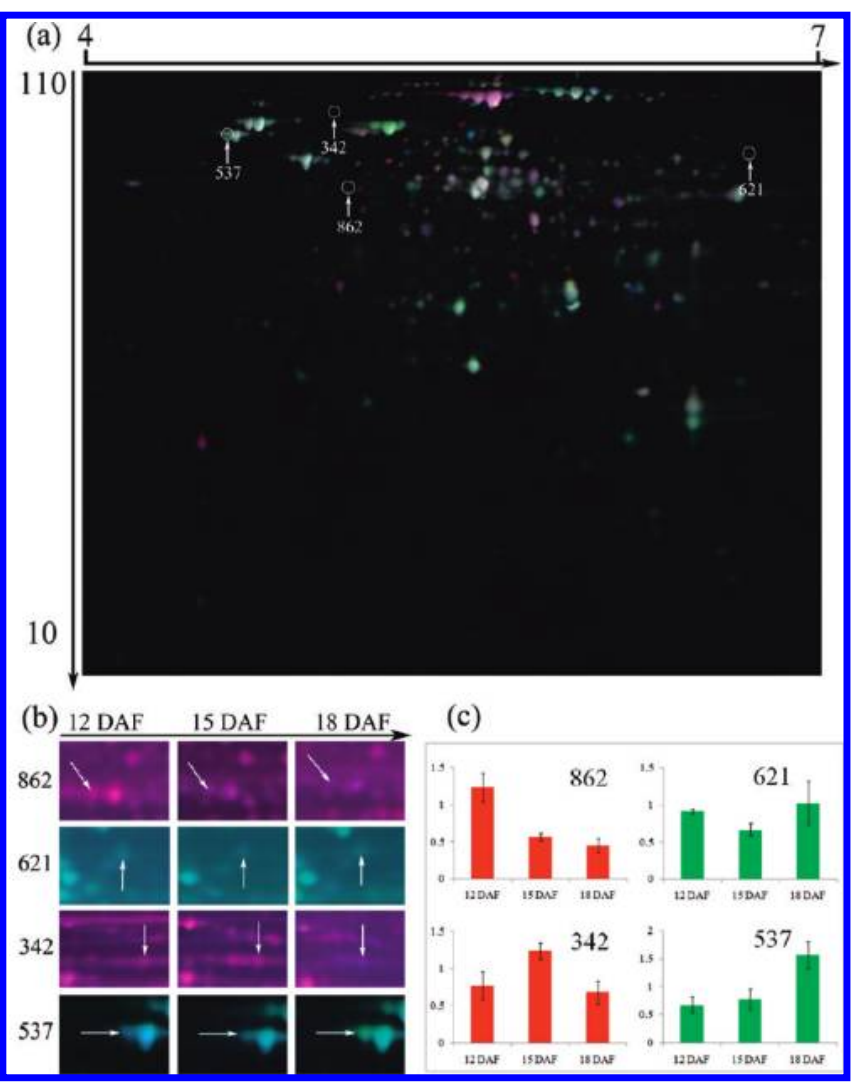

Figure 4. Representative 2D-DIGE image of endosperm proteins. (a) Representive image (image 1, all images shown in Supplemental Figure S1). Internal standard and proteins at 18 and 12 DAF were labeled by Cy2 (blue), Cy3 (green), and Cy5 (red), respectively. Four differentially expressed protein spots are exemplified by white open circles. (b) Closeup of the 4 protein spots. (c) Expression profiles of the 4 proteins. Data are mean and standard deviation of 4 replicates. Molecular mass (in kilodaltons) and $\mathrm{p} /$ of the proteins are shown at left and top of panel a, respectively.

teins. ${ }^{16}$ Here, we used 2D-DIGE with $\mathrm{pH} 4-7$ strips to separate endosperm proteins and found about 2,300 protein spots in each image (Figure 4a, Supplemental Figure S1). Our experiment design suitable to cross comparison of images from distinct samples and quantitation of protein spots (Supplemental Figure S1; for details, see Materials and Methods) resulted in 18 independent images with 4 independent biological repeats for each sample (Supplemental Figure S1).

DeCyder 6.5 analysis with ANOVA revealed 396 spots with significantly changed expression during the developmental process $(P<0.05)$ and with Student's $t$ test, 502 spots $(P<0.05)$. Finally, correction for false discovery rate confirmed 322 spots with statistically significant differential expression in the 3 distinct endosperm samples (all by ANOVA and Student's $t$ test, $P<0.05$ ) (Supplemental Table S1, examples of spots shown in Figure 4).

Identification of Differentially Expressed Proteins. MS and MS/MS analyses led to the identification of 298 spots (Supplemental Table S2, Figure S2, Figure S3). Of the 298 spots, 279 contained a single protein each, and the remaining 19 had 2 proteins each. In total, we obtained 317 identities, representing 187 unique proteins (Supplemental Table S2). Using a part and/ or an instance of the parent of gene ontology terms and the scheme for functional category classification used for rice seed proteins, ${ }^{16}$ we classified these proteins into 8 functional 


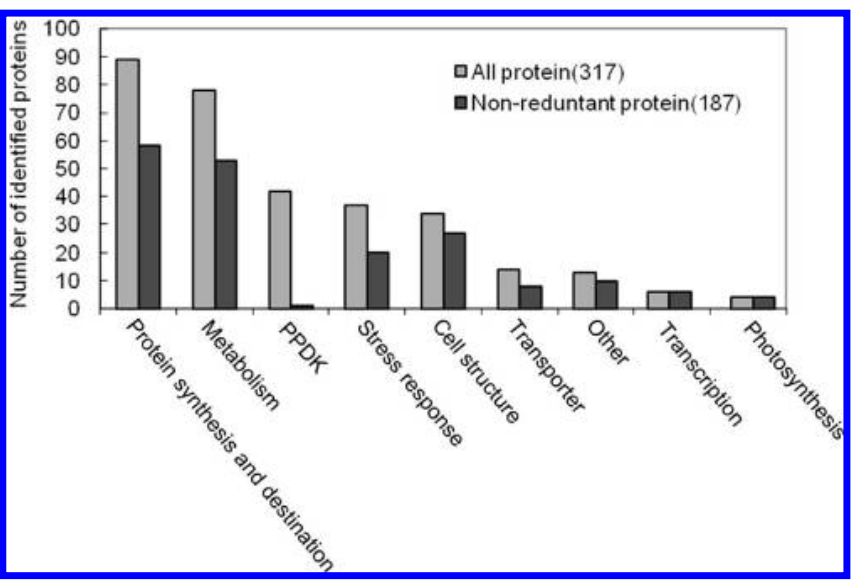

Figure 5. Functional classification of the identified differentially expressed proteins. Columns show distribution of all 317 proteins (identities, gray) and 187 unique proteins (black) in different groups. The raw data are in Supplemental Table S2.

categories (Figure 5, Supplemental Table S2). Proteins without gene ontology terms in the database and those not clearly assigned to the above 8 categories were grouped as "other".
The analysis revealed $51 \%$ of the 317 identities implicated in 2 largest functional groups: protein synthesis/destination (26\%) and metabolism (25\%), and the remaining $49 \%$ were involved in the other 7 groups (Figure 5). The results suggested that active reserve accumulation and starch granule packaging require a coordinated action of multiple metabolism or cellular processes, with protein synthesis/destination and metabolism being overrepresented in these differentially expressed proteins.

To analyze in detail the dynamic changes among different processes of protein synthesis/destination and metabolism, we further classified protein synthesis/destination-related proteins into 4 subcategories: protein synthesis, protein folding, protein modification, and proteolysis. Proteins related to metabolism were further assigned into 8 subcategories: glycolysis, tricarboxylic acid (TCA) cycle, alcoholic fermentation, starch synthesis, amino acid metabolism, nucleotide metabolism, lipid/ sterol metabolism, and secondary metabolism (Table 1, Supplemental Table S2). These subcategories provide more detailed information about changes in protein synthesis/ destination and metabolism in the storage phase.

Differentially Expressed Proteins Are Closely Related to Redox Regulation. Much evidence shows that a changeable redox potential is required for a series of cellular and devel-

Table 1. Hierarchical Clusters of Differentially Expressed Proteins and Distribution of the Proteins Involved in Each Category or Subcategory in Different Clusters ${ }^{a}$

\begin{tabular}{|c|c|c|c|c|c|c|c|}
\hline Categories or subcategories & & & & $\begin{array}{lll}c 3: & 149 \\
& & \\
& \end{array}$ & Total & PTTS & PTTs/Total \\
\hline 01 Metabolism & 11 & 11 & 9 & 30 & 61 & 46 & $75 \%$ \\
\hline 01.01 Glycolysis (or gluconeogenesis) & 3 & 3 & 0 & 9 & 15 & 12 & $75 \%$ \\
\hline 01.02 TCA cycle & 0 & 0 & 0 & 3 & 3 & 3 & $100 \%$ \\
\hline 01.03 Alcoholic fermentation & 0 & 1 & 0 & 1 & 2 & 2 & $100 \%$ \\
\hline 01.03 Starch metabolism & 2 & 1 & 5 & 9 & 17 & 16 & $94 \%$ \\
\hline 01.04 Lipid and sterol metabolism & 1 & 2 & 0 & 2 & 5 & 1 & $20 \%$ \\
\hline 01.05 Amino acid metabolism & 3 & 3 & 4 & 3 & 13 & 10 & $77 \%$ \\
\hline 01.06 Nucleotide metabolism & 1 & 1 & 0 & 1 & 3 & 0 & $0 \%$ \\
\hline 01.07 Secondary metabolism & 1 & 0 & 0 & 2 & 3 & 1 & $33 \%$ \\
\hline 02 Protein synthesis and destination & 15 & 14 & 11 & 41 & 81 & 48 & $59 \%$ \\
\hline 02.01 Protein synthesis & 4 & 3 & 2 & 2 & 11 & 8 & $73 \%$ \\
\hline 02.02 Protein folding & 8 & 8 & 6 & 19 & 41 & 37 & $90 \%$ \\
\hline 02.03 Proteolysis & 3 & 3 & 3 & 13 & 22 & 2 & $9 \%$ \\
\hline 02.04 Protein modification & 0 & 0 & 0 & 7 & 7 & 1 & $14 \%$ \\
\hline 03 PPDK & 30 & 2 & 5 & 0 & 37 & 37 & $100 \%$ \\
\hline 04. Cell structure & 12 & 5 & 2 & 8 & 27 & 15 & $56 \%$ \\
\hline 04.01 Cell growth/division & 11 & 4 & 1 & 2 & 18 & 14 & $78 \%$ \\
\hline 04.02 Cell wall & 1 & 1 & 1 & 6 & 9 & 1 & $11 \%$ \\
\hline 05 Transporter & 0 & 1 & 0 & 12 & 13 & 9 & $69 \%$ \\
\hline 06 Transcription & 2 & 1 & 0 & 3 & 6 & 1 & $17 \%$ \\
\hline 07 Stress response & 8 & 4 & 4 & 23 & 39 & 21 & $54 \%$ \\
\hline 08 Photosynthesis & 1 & 0 & 0 & 1 & 2 & 2 & $100 \%$ \\
\hline 09 Other & 3 & 1 & 1 & 8 & 13 & 3 & $23 \%$ \\
\hline Total & 82 & 39 & 32 & 126 & 279 & 182 & $65 \%$ \\
\hline
\end{tabular}

${ }^{a}$ The clusters $(\mathrm{c} 0-\mathrm{c} 3)$ are produced by GeneCluster 2.0, and raw data for the clusters are listed in Supplemental Table S3. PTTs, potential thioredoxin targets. 
Table 2. Differentially Expressed Proteins Involved in Biomacromolecule Metabolism ${ }^{a}$

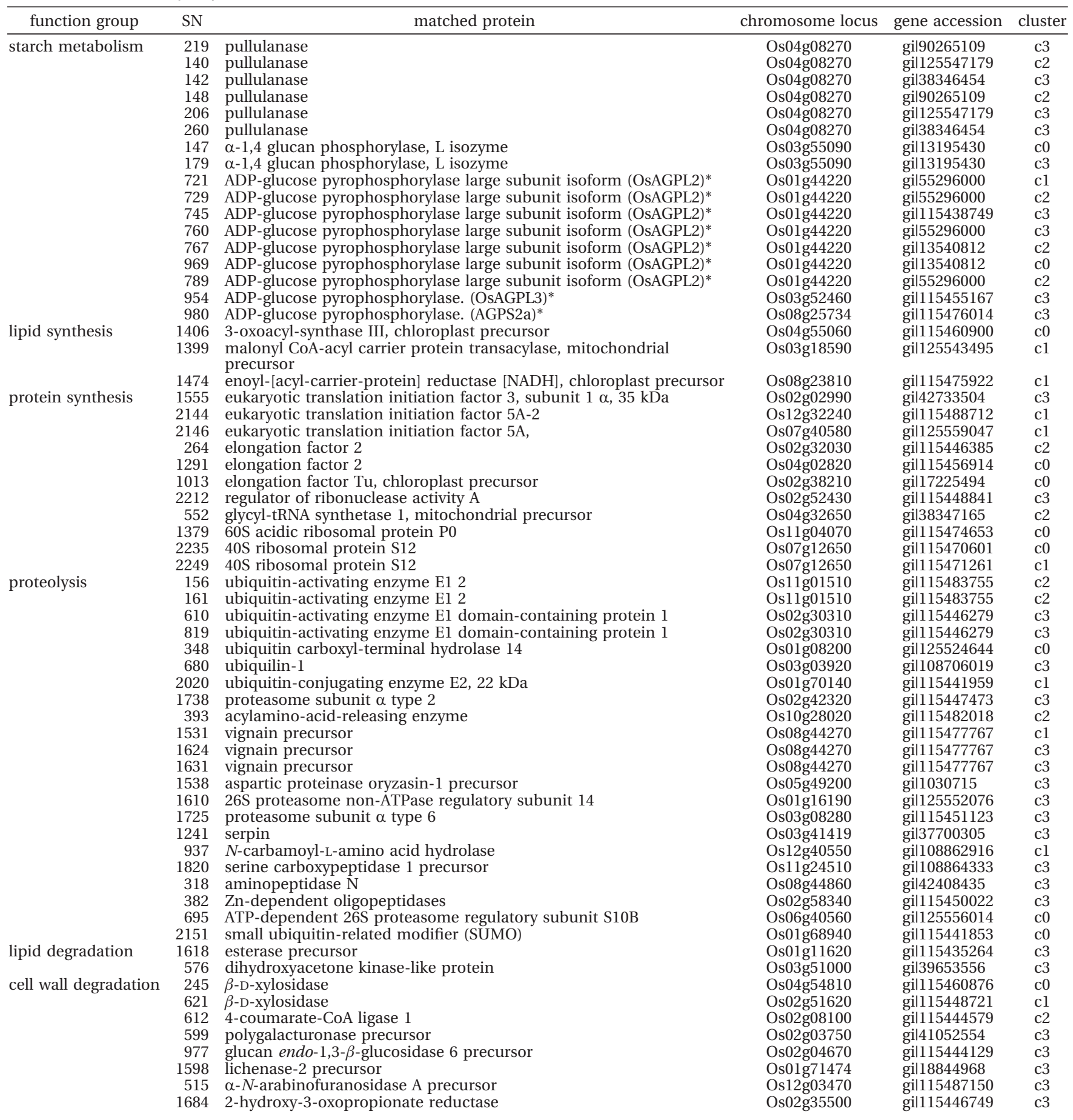

${ }^{a} \mathrm{SN}=$ protein spot number. ${ }^{*}$ indicates the gene name described in previous studies ${ }^{34,35}$ based on molecular mass and corresponding gene locus of the protein. The details are in Supplemental Table S3.

opmental events, including cereal seed development and germination. ${ }^{13}$ The proteins sensitive to redox potential, termed potential thioredoxin targets (PTTs), have been intensively investigated. ${ }^{13}$ Here, we screened possible PTTs from the differentially expressed proteins (DEPs) by use of a known PTT database $^{13,28-33}$ (Supplemental Table S3). About two-thirds of the DEPs matched known PTTs. Considering the incomplete status of known PTTs, the number of PTTs in DEPs may be more than the present prediction.

The possible PTT proteins showed heterogeneous distribution in different categories or subcategories (Table 1). For example, more than $70 \%$ of the DEPs were possible PTTs in the categories of metabolism, protein synthesis/destination, cell structure, cell growth/division, pyruvate phosphate dikinase (PPDK) and photosynthesis and in the subcategories of metabolism (glycolysis, TCA cycle, alcoholic fermentation, starch metabolism, amino acid metabolism) and protein synthesis/ destination (protein synthesis, protein folding). Less than $20 \%$ of proteins in the subcategories of nucleotide metabolism, lipid/sterol metabolism, and proteolysis were possible PTTs.

Hierarchical Clustering Analysis. To observe the expression patterns of proteins involved in each functional category 


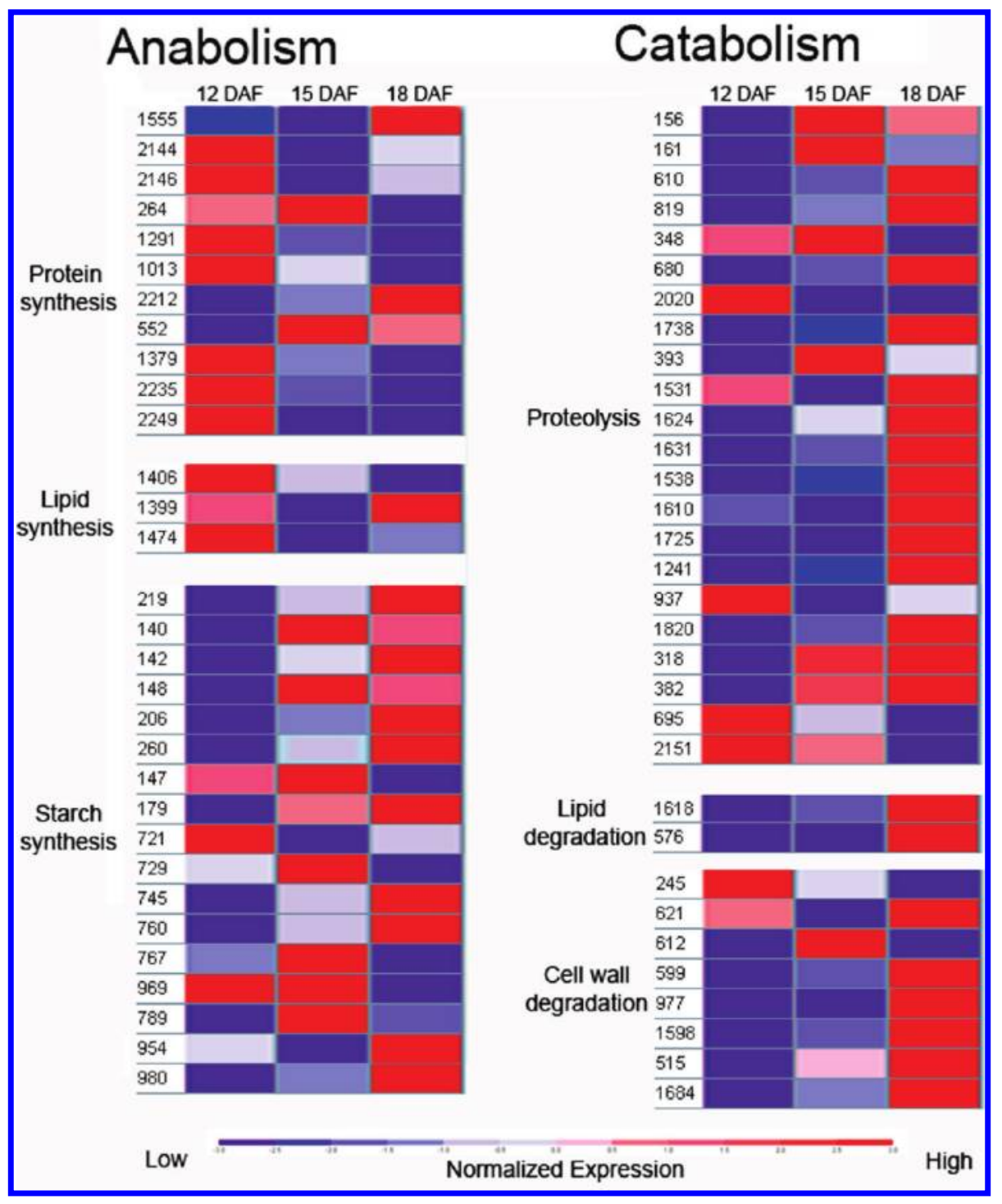

Figure 6. Expression features of proteins involved in biomacromolecule metabolism analysis. The figure was created with GeneCluster 2.0 with manual legends. The numbers represent the protein number. Details are in Table 2 and Supplemental Table S3.

(subcategory) during endosperm development, we performed cluster analysis of the 279 identities, with the exclusion of the 19 spots with 2 identities each, and revealed 4 expression patterns (clusters c0, c1, c2, and c3) (Table 1, Supplemental Table S3). The largest cluster was c3, of 126 proteins, whose abundance increased linearly from 12 to 18 DAF. The second largest cluster was c0, of 82 proteins, whose level decreased from 12 to 18 DAF. Proteins in c1 (39) and c2 (32) were downregulated and upregulated, respectively, at 15 DAF.

From biochemical functional information of proteins listed in the Protein Knowledgebase (UniprotKB/Swiss-Prot), 68 DEPs appeared to function in catalyzing a biochemical reaction by consuming energy (Supplemental Table S4). These proteins showed significantly different distribution in the 4 expression patterns (clusters) as compared with all DEPs ( $\chi^{2} 3$ DF, 44.11, $P<0.01$ ). They showed preferential downregulation (37 in cluster c0 vs 11 in cluster c3) (Supplemental Table S5), which suggests that energy-consuming reactions were slowed down in endosperm from 12 to 18 DAF. Furthermore, the digital profiles of the DEPs, established as described by Xu et al., ${ }^{16}$ showed a significant positive correlation with changed ATP content, which decreased greatly from 12 to 18 DAF (Figure 2c) ( $r=0.99995, P<0.01$; Supplemental Table S5). These data suggest that metabolism or cellular processes are switched to a state consuming relatively little energy during the starch and protein accumulation. However, the energy consumers (DEPs) with different molecular function differed in expression profiles. The kinases (Supplemental Table S4), the main energy consumers, were distributed in c0, whereas transferases were in only c2 and c3 (none in c0 or c1). All these transferases were ADP-glucose pyrophosphorylases (AGPases), the key enzyme channeling carbon solutes to starch synthesis. ${ }^{4}$

Expression Features of Proteins Related to Main Biomacromolecules. Starch is a type of preferential reserve in rice endosperm. To understand the coordinated action of starch synthesis and other biomacromolecule metabolisms during the starch and protein accumulation, we focused on the expression features of DEPs involved in the metabolism of main biomacromolecules (carbohydrates, protein, and lipid) (Table 2, Figure 6). Protein synthesis machine components, including $40 \mathrm{~S}$ and $60 \mathrm{~S}$ ribosomal proteins and most of the initiation or elongation factors (4 of 6), were greatly downregulated. By contrast, most proteolysis-related proteins (16 of 22) were markedly upregulated. This finding suggests that protein metabolism is switched 


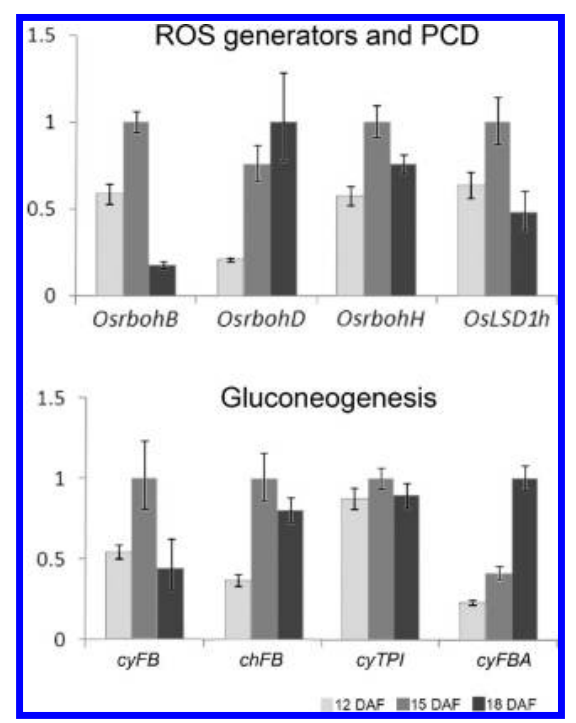

Figure 7. Expression profiles of genes involved in ROS generation, PCD, and gluconeogenesis. The expression profiles of genes in endosperm from 12 to 18 DAF were analyzed by real-time quantitative RT-PCR. OsrbohB and homologues of OsrbohD and OsrbohH were identified as ROS generators in rice (OsrbohB) and in Arabidopsis (rhobD and H). ${ }^{35,36}$ OsLSD1h is a rice homologue close to Arabidopsis LSD1, which is a ROS-responsive gene and functions in triggering PCD. ${ }^{36}$ Genes involved in the gluconeogenesis pathway are those encoding cytosolic type (cyFB) and chlotoplast type (chFB) fructose-1,6-bisphosphatases, triosephosphate isomerase (cytosolic, cyTPI), and fructose-bisphosphate aldolase (cytosolic, cyFBA). The raw data are in Supplemental Table S6.

from anabolism to catabolism. Similarly, lipid metabolism appeared to prefer catabolism because lipid synthesis proteins (spots 1399, 1406, and 1474) were downregulated and lipid degradation-related proteins were upregulated (spots 576, 1618). Meanwhile, the identified proteins involved in the hydrolysis of polysaccharides (spots 515, 599, 612, 977, 1598, and 1684), the components of the primary cell wall of cereal seeds, ${ }^{36}$ were greatly upregulated. In coordination with these changes, starch synthesis-related proteins were greatly upregulated after 12 DAF. The result suggested that the biomacromolecules, except starch, experienced the conversion from anabolism to catabolism, and the conversion may be important for the efficient synthesis of starch.

Expression Analysis of Genes Related to ROS Generation and Gluconeogenesis. The rice endosperm experienced an $\mathrm{H}_{2} \mathrm{O}_{2}$ burst and PCD during the starch and protein accumulation phase (see above). Studies have showed that respiratory burst oxidase homologue (rboh) is the main generator of ROS in plants, ${ }^{37}$ and LSD1 (lysine-specific histone demethylase 1), a gene responsive to ROS, functions in triggering PCD in Arabidopsis. ${ }^{38}$ We analyzed the expression of 3 rice rboh genes (OsrbohB, OsrbohD and OsrbohH) and the rice LSD1 homologue $(O S L S D 1 h)$ in 12 to 18 DAF endosperm using real-time quantitative RT-PCR (Figure 7, Table S6). These 3 rboh genes showed upregulated expression at 15 DAF (2 of 3) and 18 DAF (1 of 3), which was temporally in line with the $\mathrm{H}_{2} \mathrm{O}_{2}$ burst observed by DAB staining and $\mathrm{H}_{2} \mathrm{O}_{2}$ quantitative assay (Figure 1). Correspondingly, OsLSD1 $h$ was expressed at the highest level at 15 DAF. Together, these results indicated the connection between $\mathrm{H}_{2} \mathrm{O}_{2}$ generators, $\mathrm{H}_{2} \mathrm{O}_{2}$ burst and PCD in rice endosperm. In addition, our proteomics analysis identified the chloroplast type (spot 1046) of fructose-1,6-bisphosphatase, an enzyme of the gluconeogenesis pathway. We further evaluated the change in gluconeogenesis by analyzing the gene expression of the cytoplasm-type fructose-1,6-bisphosphatase and other enzyme proteins involved in the pathway. Genes encoding these proteins showed increased expression at 15 DAF (3 of 4) (Figure 7), which was in agreement with the expression profile of the chloroplast-type fructose-1,6-bisphosphatase observed in proteomics analysis. Therefore, gluconeogenesis was perhaps activated under an $\mathrm{H}_{2} \mathrm{O}_{2}$ burst background.

\section{Discussion}

Progression of Starch Granule Packaging Specifies the Fate of Starchy Endosperm Cells. The starchy endosperm functions as a storage tissue but is fated to die before the maturation of seeds in cereal plants. ${ }^{5}$ Previous studies of maize and barley have revealed that starch first is deposited in the central endosperm and then spreads toward the periphery during development, ${ }^{6,39}$ and endosperm cell death closely follows the pattern of starch deposition in maize. ${ }^{6}$ We revealed that the developing rice endosperm displays a pattern of starch accumulation and cell death similar to that in barley and maize. This finding suggests that a common position cue may specify the fate of starchy endosperm cells during development, at least in rice, maize, and barley. However, the identity of the position cue is unknown. We found that the completion of starch granule packaging was concurrent spatially and temporally with position-dependent senescence or death of starchy endosperm cells. First, the complete packaging of starch granules appeared in the inner part at $15 \mathrm{DAF}$, where nonviable cells were observed. Second, nonviable cells spread outward from the inner part as starch granule packaging progressed during development. Third, the 3 genes of the $\mathrm{H}_{2} \mathrm{O}_{2}$ (ROS) generators were expressed at high levels at 15 DAF (2 of 3), and a ROS burst was observed at 15 DAF (Figures 1 and 7). Fourth, ROS is considered one of key elements initiating cell death, ${ }^{7,25}$ and we found the rice homologue of LSD1, which in Arabidopsis was responsive to ROS and functions in triggering $\mathrm{PCD},{ }^{38}$ greatly upregulated at 15 DAF (Figure 7). These data suggest that the state of starch granule packaging seems be one of important position cues specifying the fate of starchy endosperm cells, and the mechanism may be important to ensure that nutrient solutes are transported first into the innermost parts of endosperm for efficient starch accumulation from inner to outer parts.

Little detailed data exist to clarify the association of the state of starch granule packaging and the generation of ROS and starchy endosperm cell death. Several observations showed that osmotic stress in plant tissues led to the ROS burst and eventually PCD. ${ }^{5}$ We showed decreased water content during the development phase of rice endosperm, and the endosperm containing fully packed starch granules had a desiccated appearance (Figure 2). These findings, in combination with greatly increased level of the desiccation protein PCC13-62 (spots 1593, 1635, and 1614, Supplemental Table S3), suggest that a sharp change in osmotic potential caused by starch deposition may involve the onset of starchy endosperm cell death possibly via ROS and/or other unknown molecules at the completion of starch granule packaging.

Importance of Redox Regulation in Endosperm Development and Starch Accumulation. ROS are key signaling molecules and are controlled by the antioxidative system to a level suitable to maintain cellular or metabolism processes for 


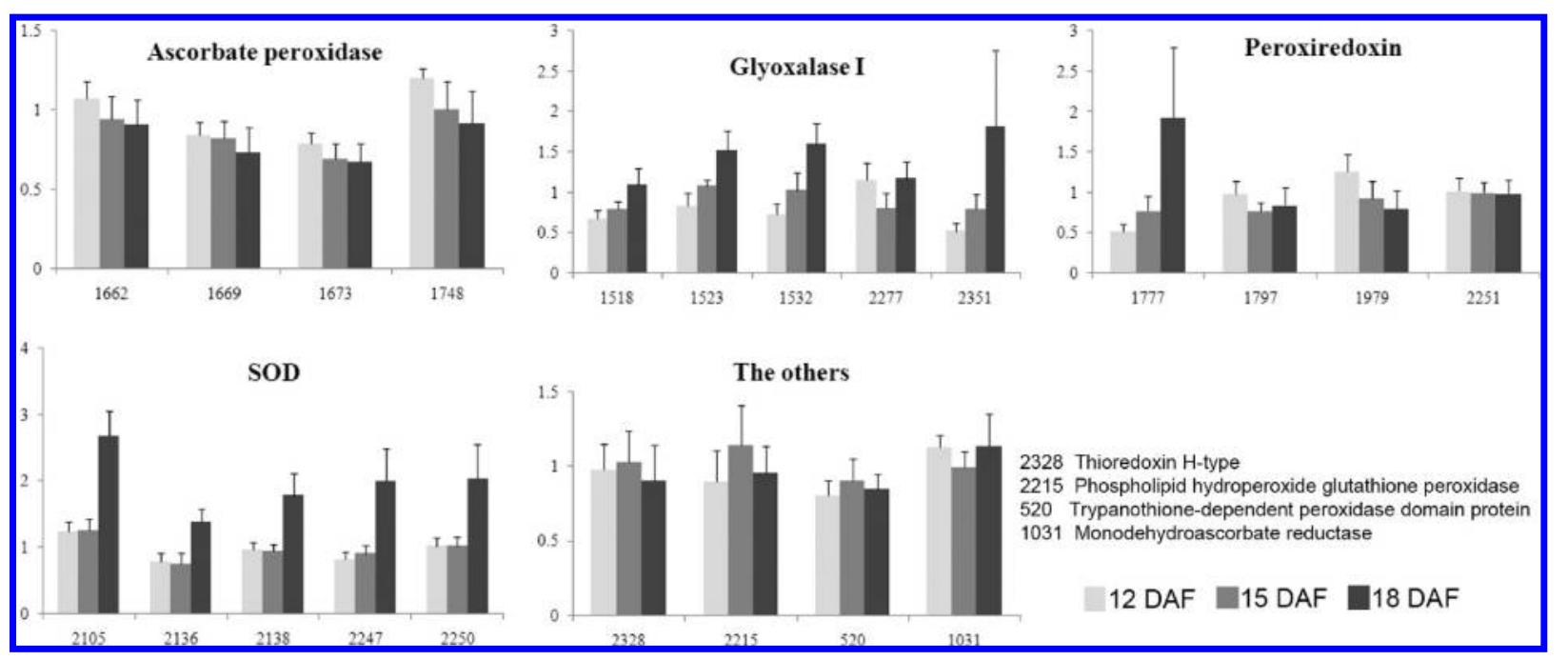

Figure 8. Expression profiles of antioxidants. The vertical axis shows the relative volume of each protein. Numbers in the horizontal axis represent identity numbers of identified proteins, which are in Supplemental Table S3.

normal growth and development. ${ }^{11}$ The signal mechanism functions in regulation of photosynthetic carbon metabolism and partitioning in leaves and potato tubers. ${ }^{14}$ In the development phase of rice endosperm, the $\mathrm{H}_{2} \mathrm{O}_{2}$ burst occurred at 15 DAF at the completion of starch granule packaging in the inner endosperm part (also see discussion above). As well, 22 ROS scavenging-related proteins displaying differential expression (Figure 8) included the known thiol buffers thioredoxin (Trx) ${ }^{11}$ and key ROS scavengers GSH peroxidase (GPX), ascorbate peroxidase (APX), mondehydroascorbate reductase (MDAR), ${ }^{38}$ peroxiredoxin (Prx), superoxide dismutase (SOD), ${ }^{13}$ phospholipid hydroperoxide glutathione peroxidase (PHGPX), ${ }^{39}$ and trypanothione-dependent peroxidase (TDP). ${ }^{40}$ Among them, Trx (spot 2328), PHGPX (spot 2215), GPX (spot 2215), and TDP (spot 520) were expressed at the highest levels at 15 DAF. All SOD proteins (spots 2105, 2136, 2138, and 2237) and Prx (spot 1777) were at the highest level at $18 \mathrm{DAF}$; all APX proteins (spots $1662,1669,1673$, and 1748) were decreased in level from 12 to 18 DAF; and MADR (spot 1031) was expressed at higher level at 12 and 18 DAF than at 15 DAF. Four of the 5 identified glyoxalase I isoforms showed increased expression. Glyoxalase I, in combination with glyoxalase II, catalyzes the detoxification of methylglyoxal (MG) to lactate by the GSH cycle. ${ }^{41} \mathrm{MG}$ is a transition-state intermediate of the triose-phosphate step of the glycolysis (or gluconeogenesis) pathway under normal conditions and is increased in level in response to osmotic stress in plants. ${ }^{42}$ Tobacco plants overexpressing glyoxalase are resistant to increased level of MG and maintain a highly reduced glutathione level under salinity stress. ${ }^{42}$ APX, MADR, and GPX are key enzymes detoxifying $\mathrm{H}_{2} \mathrm{O}_{2}$ to $\mathrm{H}_{2} \mathrm{O}$ by ascorbate and GSH. ${ }^{9}$ The identified TRX (spot 2328) was the $h$ type TRX and together with glutathione is a key thiol buffer that functions mainly in reducing levels of disulfides. ${ }^{11}$ Prx mediates the detoxification of peroxides by an intermolecular thioldisulfide transition. ${ }^{43}$ The diverse expression patterns of these ROS scavenging-related proteins suggests that a coordinated redox reaction is important in the endosperm reserve storage phase.

Several studies have shown that the development of cereal seeds involves a classic oxidative process, ${ }^{12,13}$ and redox regulation is essential for the synthesis, maturation, and stability of storage proteins during seed maturity. ${ }^{12,33,44}$ The respective up- and downregulation of SOD and APX proteins in the rice endosperm (Figure 8) was consistent with the expression profiles of the 2 protein types in wheat seeds, which are involved in desiccation of cereal seeds. ${ }^{45,46}$ Aberrantly reduced expression of SOD in viviparous 1 kernels of maize led to a premature induction of endosperm PCD. ${ }^{5}$ Our proteomic results showed that several or most of the proteins involved in diverse metabolism/cellular processes including energy metabolism and starch synthesis were coexpressed at a significantly similar level with SOD, APX, or glyoxalase I (Supplemental Table S7). Furthermore, in the rice endosperm, most DEPs were PTTs (Table 1, Supplemental Table S3) involved in different metabolism or cellular processes, with preference for the TCA cycle, glycolysis, alcoholic fermentation, starch synthesis, PPDK, photosynthesis, and protein folding. These findings are in line with an early notion that redox modulation plays vital roles in energy metabolism ${ }^{10,14}$ and in regulation of carbon storage and partitioning in plant tissues such as tubers and cereal seeds. ${ }^{13,14}$ Together with the feature of preferential starch synthesis and decreased generation of ATP (Figure 2c) in the developing endosperm, these lines of evidence suggest that a coordinated action by redox and other unknown modulations is required for efficient partitioning of nutrient solutes from imported sucrose and remobilized carbon (see below) into starch synthesis possibly by affecting stability and activity of enzyme proteins. ${ }^{13}$ For example, the key enzyme AGPase is closely regulated by redox regulation. ${ }^{14}$ However, the detailed function of the most PTTs and antioxidants under redox regulation remains to be determined.

Nutrition Remobilization during Endosperm PCD Is Required for Efficient Starch Accumulation. The death of starchy endosperm cells is proposed to be required for efficient secretion of aleurone- or scutellum-derived hydrolases into the endosperm to mobilize rapid storage materials and support early postembryonic growth during germination. ${ }^{13,47}$ The importance of the default cell program in accumulation of reserve materials by nutrition remobilization is not clear. Our results showed that the position cue-dependent senescence or death of starchy endosperm cells may be important in nutrition remobilization for efficient accumulation of reserve materials (Figure 6, Figure 9). With the completion of starch accumulation and packaging, the endosperm experienced a remarkable 


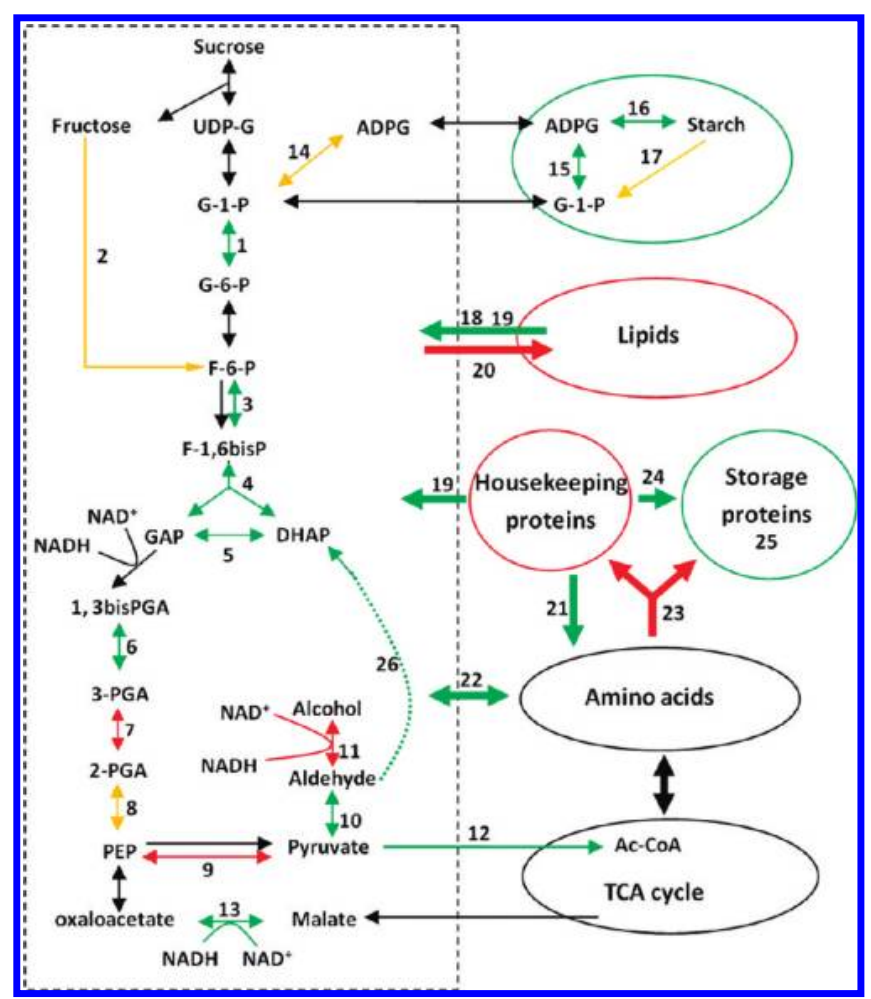

Figure 9. Simplified schematic representation of carbon flux at the storage phase of endosperm development. Fine line arrows represent reaction direction catalyzed by the corresponding enzymes. Bold line arrows show putative direction of carbon flux. Green indicates upregulation, red downregulation, and orange unclear expression, although related enzymes are identified, and black indicates unknown changed process because of no identification of specific enzyme proteins. Numbers represent the serial number of reactions and the differentially expressed enzyme proteins implicated in corresponding reactions: 1, Phosphoglucomutase. 2, Fructokinase-2. 3, Fructose-1,6-bisphosphatase. 4, Fructose-bisphosphate aldolase. 5, Triosephosphate isomerase. 6, Phosphoglycerate kinase. 7, 2,3-bisphosphoglycerate-independent phosphoglycerate mutase. 8, Enolase. 9, Pyruvate orthophosphate dikinases. 10, Pyruvate decarboxylase isozyme 2. 11, Alcohol dehydrogenase 1. 12, Pyruvate dehydrogenase E1, acetyl-coenzyme A synthetase, and dihydrolipoyl dehydrogenase. 13, Malate dehydrogenase, cytoplasmic. 14, ADP-glucose pyrophosphorylase large subunit (OsAGPL2). 15, ADP-glucose pyrophosphorylase large subunit 3 (OsAGPL3) and ADP-glucose pyrophosphorylase small subunit (AGPS2a). 16, Pullulanase. 17, $\alpha-1,4$ glucan phosphorylase. 18, Lipiddegradation proteins: esterase and 3,4-dihydroxy-2-butanone kinase. 19, Acid phosphatase and lysosomal $\alpha$-mannosidase. 20, Lipid-synthesis proteins: ketoacyl-acyl carrier protein synthase III, malonyl CoA-acyl carrier protein transacylase, and enoyl-[acyl-carrier-protein] reductase. 21, Proteolysis-related proteins. 22, Dihydrolipoyllysine-residue succinyltransferase. 23 , Proteins involved in protein synthesis. 24 , Protein disulfide isomerase. 25, Storage proteins: desiccation-related protein PCC13-62 and germin-like protein. 26, Glyoxalase. Detailed information on isoforms and expression patterns of the proteins are in Supplemental Table S3. UDP-G, uracil-diphosphate glucose; G-1-P, glucose-1-phosphate; G-6-P, glucose-6phosphate; F-6-P, fructose-6-phosphate; F-1,6bisP, fructose1,6-bisphosphate; GAP, glyceraldehyde-phosphate; 1,3bisPGA, 1,3-bisphosphoglycerate; 3-PGA, glyceraldehyde 3-phosphate; 2-PGA, glyceraldehyde 2-phosphate; DHAP, dihydroxyacetone phosphate; PEP, phosphoenolpyruvate; NADH, nicotinamide adenine dinucleotide; Ac-CoA, acetyl coenzyme A; ADPG, adenosine diphosphoglucose. metabolism conversion from anabolism to catabolism for protein, lipid, and polysaccharide components of the primary cell wall (Figure 6, also see Results). The increased preference for catabolism of these biomacromolecules during the process may lead to remobilization of more free amino acids, additional carbohydrates and lipids. Correspondingly, from 12 to $18 \mathrm{DAF}$, the key enzymes involved in nutrition remobilization were increased in level; examples are acid phosphatase 1 (spots 1524, 1527, and 1630) and $3^{\prime}\left(2^{\prime}\right), 5^{\prime}$-bisphosphate nucleotidase (spots 1114 and 1134), and $\alpha$-mannosidase (spots 485, 492 and 1257) (Figure 9, Supplemental Table S3). The former 2 types of enzyme proteins play important roles in the remobilization of $\mathrm{Pi}$ and sulfur, ${ }^{48,49}$ whereas the latter functions in the breakdown of glycoproteins and $\mathrm{N}$-linked glycans for remobilization of carbohydrates and free amino acids. ${ }^{50,51}$ In addition, the dihydrolipoyllysine-residue succinyltransferase (spots 973 and 986), playing a key role in the conversion between amino acids and carbohydrates, showed upregulated expression, which suggests the active conversion between amino acids and carbohydrates.

Corresponding to increased catabolism of the biomacromolecules described above, upregulated proteins (c3) were all isoforms of luminal-binding protein 3 (spots 418, 423, 425, 447 and 966) and protein disulfide isomerase (PDI) (OsPDIL1-4, spots 523, 527, 537, 596, and 601) (Supplemental Table S3), considered markers of storage protein synthesis, ${ }^{52}$ and 2 cysteine synthases (spots 1351 and 1561), which supply necessary residues for disulfide bridge formation of PDI and for sulfur to storage proteins. ${ }^{53}$ Therefore, storage proteins may be the destination of remobilized nitrogen and sulfur. Consistent with this notion, previous research revealed that the lack of the 26$\mathrm{kDa}$ globulin gene markedly increased the level of free amino acids in rice grains. ${ }^{54}$

In general, the carbon skeleton from catabolism of the biomacromolecules is partitioned mainly via the glycolysis pathway for resynthesis of target products and/or for energy production. ${ }^{55}$ The cereal endosperm is a typical hypoxia tissue, and hypoxia stress increases with endosperm development. ${ }^{56}$ Proteomic and transcriptomic studies in maize and rice have suggested that ATP generation is inhibited with the increasing hypoxia stress as seeds (endosperm) develop. ${ }^{16,57,58}$ Correspondingly, our observation revealed a steep decrease in ATP content during the endosperm development (Figure 2). In the rice endosperm, the glycolysis enzyme proteins involved in reactions leading to generation of 3-phosphoglycerate (3-PGA) were increased in level, whereas the enzyme proteins involved in conversion of 3-PGA to pyruvate were decreased in level from 12 to 15 DAF, which suggests increased generation of 3-PGA, a classic allosteric activator of AGPase that channels the incoming carbon source toward synthesis of storage starch. ${ }^{14}$ AGPase proteins showed increased expression in the development phase (Figure 6). Consistently, the upregulated expression of fructose-1,6-bisphosphatase (spot 1046) and cytoplasmic malate dehydrogenase (spot 1436), the key enzymes of gluconeogenesis, ${ }^{59}$ along with upregulated expression of genes encoding other key enzyme proteins of the pathway (Figure 7), was similar to that of starch synthesis-related enzymes, including AGPase (Figure 6). Study of Arabidopsis seeds showed that the gluconeogenesis pathway is essential for mobilizing the carbon source from storage lipids. ${ }^{60}$ Together, these results suggest that in addition to the importance in increasing carbon flux into starch synthesis, ${ }^{16}$ the glycolysis pathway may have roles in repartitioning the carbon skeleton from degradation of pro- 
teins, lipids and other biomolecules during endosperm development. As well, the remobilized carbon skeleton may be partitioned mainly into synthesis of storage starch by the gluconeogenesis pathway, and the remobilization of nutrients involves a concerted protein network integrating distinct metabolisms.

In summary, we systemically analyzed the proteomic, physiological, and cellular characteristics of rice endosperm at storage phases from 12 to 18 DAF. Starch granule packaging appeared to be complete first in the inner and then outer parts of the endosperm, and the spatiotemporal pattern of starch granule packaging was concurrent with the PCD of endosperm. Nutrition remobilization during endosperm PCD is required for efficient starch accumulation. Our results also suggest that $\mathrm{H}_{2} \mathrm{O}_{2}$-antioxidant interaction is required for the coordinated actions of cell death and carbon flux into reserve accumulation by mediating a protein network.

Acknowledgment. This work was supported by the Chinese Ministry of Science and Technology (grant nos 2006CB910105 and 2005CB120804).

Supporting Information Available: Figure S1, experiment design for Cydye label and DIGE images.

Figure S2, PMF-based Protein identifications.

Figure S3, MS/MS identifications.

Table S1, expression profile data for 922 protein spots present in all 18 images of 2D-DIGE analysis.

Table S2, identities of differentially expressed proteins determined by MALDI-TOF/TOF MS.

Table S3, protein distribution in hierarchical clusters and potential thioredoxin targets.

Table S4, differentially expressed proteins involved in energy consumption.

Table S5, (A) Chi-square test of the distribution of all differentially expressed proteins (DEPs) and energy-consumed DEPs; (B) centroids data for each hierarchical cluster created by GeneCluster 2.0; (C) correlation analysis of digital profile of energy-consumed DEPs and ATP content.

Table S6, real-time quantitative RT-PCR analysis of gene expression.

Table S7, correlation of expression profiles of ROS scavengers and all DEPs. This material is available free of charge via the Internet at http://pubs.acs.org.

\section{References}

(1) Olsen, O. A.; Linnestad, C.; Nichols, S. E. Developmental biology of the cereal endosperm. Trends Plant Sci. 1999, 4, 253-257.

(2) Olsen, O. Nuclear endosperm development in cereals and arabidopsis thaliana. Plant Cell 2004, 16, S214-S227.

(3) Sabelli, P. A.; Larkins, B. A. The development of endosperm in grasses. Plant Physiol. 2009, 149, 14-26.

(4) James, M. G.; Denyer, K.; Myers, A. M. Starch synthesis in the cereal endosperm. Curr. Opin. Plant Biol. 2003, 6, 215-222.

(5) Young, T. E.; Gallie, D. R. Programmed cell death during endosperm development. Plant Mol. Biol. 2000, 44, 283-301.

(6) Young, T. E.; Gallie, D. R.; DeMason, D. A. Ethylene-mediated programmed cell death during maize endosperm development of wild-type and shrunken2 genotypes. Plant Physiol. 1997, 115, 737751.

(7) Hoeberichts, F. A.; Woltering, E. J. Multiple mediators of plant programmed cell death: interplay of conserved cell death mechanisms and plant-specific regulators. Bioessavs 2003, 25, 47-57.

(8) Pei, Z.; Murata, Y.; Benning, G.; Thomine, S.; Klusener, B.; Allen, G.; Grill, E.; Schroeder, J. Calcium channels activated by hydrogen peroxide mediate abscisic acid signalling in guard cells. Nature 2000, 406, 731-734.
(9) Apel, K.; Hirt, H. Reactive oxygen species: metabolism, oxidative stress, and signal transduction. Annu. Rev. Plant Biol. 2004, 55, 373-399.

(10) Ying, W. H. NAD(+)/ NADH and NADP(+)/NADPH in cellular functions and cell death: Regulation and biological consequences. Antioxid. Redox Signaling 2008, 10, 179-206.

(11) Foyer, C. H.; Noctor, G. Redox homeostasis and antioxidant signaling: a metabolic interface between stress perception and physiological responses. Plant Cell 2005, 17, 1866-1875.

(12) Gobin, P.; Ng, P. K. W.; Buchanan, B. B.; Kobrehel, K. Sulfhydryldisulfide changes in proteins of developing wheat grain. Plant Physiol. Biochem. 1997, 35, 777-783.

(13) Buchanan, B. B.; Balmer, Y. Redox regulation: a broadening horizon. Annu. Rev. Plant Biol. 2005, 56, 187-220.

(14) Geigenberger, P.; Kolbe, A.; Tiessen, A. Redox regulation of carbon storage and partitioning in response to light and sugars. L. Exp. Bot. 2005, 56, 1469-1479.

(15) Lim, P. O.; Kim, H. J.; Gil Nam, H. Leaf senescence. Annu. Rev. Plant Biol. 2007, 58, 115-136.

(16) Xu, S. B.; Li, T.; Deng, Z. Y.; Chong, K.; Xue, Y.; Wang, T. Dynamic proteomic analysis reveals a switch between central carbon metabolism and alcoholic fermentation in oryza sativa filling grains. Plant Physiol. 2008, 148, 908-925.

(17) Wei, C. X.; Lan, S. Y.; Xu, Z. X. Ultrastructural features of nucleus degradation during programmed cell death of starchy endosperm cells in rice. Acta Bot. Sin. 2002, 44, 1396-1402.

(18) Thordal-Christensen, H.; Zhang, Z.; Wei, Y.; Collinge, D. B. Subcellular localization of $\mathrm{H}_{2} \mathrm{O}_{2}$ in plants. $\mathrm{H}_{2} \mathrm{O}_{2}$ accumulation in papillae and hypersensitive response during the barleypowdery mildew interaction. Plant I. 1997, 11, 1187-1194.

(19) Bradford, M. M. A rapid and sensitive method for the quantitation of microgram quantities of protein utilizing the principle of protein-dye binding. Anal. Biochem. 1976, 72, 248-254.

(20) Barcelo-Batllori, S.; Kalko, S. G.; Esteban, Y.; Moreno, S.; Carmona, M. C.; Gomis, R. Integration of DIGE and bioinformatics analyses reveals a role of the antiobesity agent tungstate in redox and energy homeostasis pathways in brown adipose tissue. Mol. Cell Proteomics 2008, 7, 378-393.

(21) Benjamini, Y.; Hochberg, Y. On the adaptive control of the false discovery rate in multiple testing with independent statistics. $J$. Educ. Behav. Stat. 2000, 25, 60-83.

(22) Dai, S.; Li, L.; Chen, T.; Chong, K.; Xue, Y.; Wang, T. Proteomic analyses of Oryza sativa mature pollen reveal novel proteins associated with pollen germination and tube growth. Proteomics 2006, 6, 2504-2529.

(23) Ishimaru, T.; Matsuda, T.; Ohsugi, R.; Yamagishi, T. Morphological development of rice caryopses located at the different positions in a panicle from early to middle stage of grain filling. Funct. Plant Biol. 2003, 30, 1139-1149.

(24) Young, T. E.; Gallie, D. R. Analysis of programmed cell death in wheat endosperm reveals differences in endosperm development between cereals. Plant Mol. Biol. 1999, 39, 915-926.

(25) Harman, D. A theory based on free radical and radiation chemistry. L Gerontol. 1956, 11, 298-300.

(26) Li, X.; Franceschi, V. R.; Okita, T. W. Segregation of storage protein mRNAs on the rough endoplasmic reticulum membranes of rice endosperm cells. Cell 1993, 72, 869-879.

(27) Bechtel, D.; Zayas, I.; Kaleikau, L.; Pomeranz, Y. Size-distribution of wheat starch granules during endosperm development. Cereal Chem. 1990, 67, 59-63.

(28) Alkhalfioui, F.; Renard, M.; Vensel, W. H.; Wong, J.; Tanaka, C. K.; Hurkman, W. J.; Buchanan, B. B.; Montrichard, F. Thioredoxinlinked proteins are reduced during germination of Medicago truncatula seeds. Plant Physiol. 2007, 144, 1559-1579.

(29) Balmer, Y.; Vensel, W. H.; Cai, N.; Manieri, W.; Schurmann, P.; Hurkman, W. J.; Buchanan, B. B. A complete ferredoxin/thioredoxin system regulates fundamental processes in amyloplasts. Proc. Natl. Acad. Sci. U.S.A. 2006, 103, 2988-2993.

(30) Balmer, Y.; Vensel, W. H.; Tanaka, C. K.; Hurkman, W. J.; Gelhaye, E.; Rouhier, N.; Jacquot, J. P.; Manieri, W.; Schurmann, P.; Droux, M.; Buchanan, B. B. Thioredoxin links redox to the regulation of fundamental processes of plant mitochondria. Proc. Natl. Acad. Sci. U.S.A. 2004, 101, 2642-2647.

(31) Marchand, C.; Le Marechal, P.; Meyer, Y.; Decottignies, P. Comparative proteomic approaches for the isolation of proteins interacting with thioredoxin. Proteomics 2006, 6, 6528-6537.

(32) Marchand, C.; Le Marechal, P.; Meyer, Y.; Miginiac-Maslow, M.; Issakidis-Bourguet, E.; Decottignies, P. New targets of Arabidopsis thioredoxins revealed by proteomic analysis. Proteomics 2004, 4, 2696-2706. 
(33) Wong, J. H.; Cai, N.; Balmer, Y.; Tanaka, C. K.; Vensel, W. H.; Hurkman, W. J.; Buchanan, B. B. Thioredoxin targets of developing wheat seeds identified by complementary proteomic approaches. Phytochemistry 2004, 65, 1629-1640.

(34) Ohdan, T.; Francisco Jr, P.; Sawada, T.; Hirose, T.; Terao, T.; Satoh, H.; Nakamura, Y. Expression profiling of genes involved in starch synthesis in sink and source organs of rice. L. Exp. Bot. 2005, 56, 3229-3244.

(35) Sikka, V.; Choi, S.; Kavakli, I.; Sakulsingharoj, C.; Gupta, S.; Ito, H.; Okita, T. Subcellular compartmentation and allosteric regulation of the rice endosperm ADPglucose pyrophosphorylase. Plant Sci. 2001, 161, 461-468.

(36) Harris, P. J.; Smith, B. G. Plant cell walls and cell-wall polysaccharides: structures, properties and uses in food products. Int.J. Food Sci. Technol. 2006, 41, 129-143.

(37) Wong, H.; Pinontoan, R.; Hayashi, K.; Tabata, R.; Yaeno, T.; Hasegawa, K.; Kojima, C.; Yoshioka, H.; Iba, K.; Kawasaki, T. Regulation of rice NADPH oxidase by binding of Rac GTPase to its N-terminal extension. Plant Cell 2007, 19, 4022-4034.

(38) Foyer, C.; Noctor, G. Redox homeostasis and antioxidant signaling: a metabolic interface between stress perception and physiological responses. Plant Cell 2005, 17, 1866-1875.

(39) Avsian-Kretchmer, O.; Eshdat, Y.; Gueta-Dahan, Y.; Ben-Hayyim, G. Regulation of stress-induced phospholipid hydroperoxide glutathione peroxidase expression in citrus. Planta 1999, 209, 469477.

(40) Alphey, M. S.; Gabrielsen, M.; Micossi, E.; Leonard, G. A.; McSweeney, S. M.; Ravelli, R. B. G.; Tetaud, E.; Fairlamb, A. H.; Bond, C. S.; Hunter, W. N. Tryparedoxins from Crithidia fasciculata and Trypanosoma brucei: photoreduction of the redox disulfide using synchrotron radiation and evidence for a conformational switch implicated in function. I. Biol. Chem. 2003, 278, 25919-25925.

(41) Dixon, D. P.; Cummins, I.; Cole, D. J.; Edwards, R. Glutathionemediated detoxification systems in plants. Curr. Opin. Plant Biol. 1998, 1, 258-266.

(42) Yadav, S. K.; Singla-Pareek, S. L.; Ray, M.; Reddy, M. K.; Sopory, S. K. Methylglyoxal levels in plants under salinity stress are dependent on glyoxalase I and glutathione. Biochem. Biophys. Res. Commun. 2005, 337, 61-67.

(43) Rouhier, N.; Gelhaye, E.; Jacquot, J. Glutaredoxin-dependent peroxiredoxin from poplar. J. Biol. Chem. 2002, 277, 13609-13614.

(44) Dupont, F. Metabolic pathways of the wheat (Triticum aestivum) endosperm amyloplast revealed by proteomics. BMC Plant Biol. 2008, $8,39$.

(45) Franca, M. B.; Panek, A. D.; Eleutherio, E. C. A. Oxidative stress and its effects during dehydration. Comp. Biochem. Phvs. A 2007, 146, 621-631.

(46) De Gara, L.; de Pinto, M.; Moliterni, V.; D’Egidio, M. Redox regulation and storage processes during maturation in kernels of Triticum durum. L. Exp. Bot. 2003, 54, 249-258.
(47) Yano, H.; Wong, J. H.; Cho, M. J.; Buchanan, B. B. Redox changes accompanying the degradation of seed storage proteins in germinating rice. Plant Cell Phvsiol. 2001, 42, 879-883.

(48) Peng, Z.; Verma, D. P. S. A rice HAL2-like gene encodes a Ca(2+)sensitive $3^{\prime}\left(2^{\prime}\right), 5^{\prime}$-diphosphonucleoside $3^{\prime}\left(2^{\prime}\right)$-phosphohydrolase and complements yeast met22 and Escherichia coli cysQ mutations. L. Biol. Chem. 1995, 270, 29105-29110.

(49) Hur, Y. J.; Lee, H. G.; Jeon, E. J.; Lee, Y. Y.; Nam, M. H.; Yi, G.; Eun, M. Y.; Nam, J.; Lee, J. H.; Kim, D. H. A phosphate starvationinduced acid phosphatase from Oryza sativa: phosphate regulation and transgenic expression. Biotechnol. Lett. 2007, 29, 829-835.

(50) Winchester, B. Lysosomal metabolism of glycoproteins. Glycobiology 2005, 15, 1R-15R.

(51) Chantret, I.; Moore, S. E. H. Free oligosaccharide regulation during mammalian protein N-glycosylation. Glycobiology 2008, 18, 210224.

(52) Kawagoe, Y.; Suzuki, K.; Tasaki, M.; Yasuda, H.; Akagi, K.; Katoh, E.; Nishizawa, N. K.; Ogawa, M.; Takaiwa, F. The critical role of disulfide bond formation in protein sorting in the endosperm of rice. Plant Cell 2005, 17, 1141-1153.

(53) Hesse, H.; Nikiforova, V.; Gakiere, B.; Hoefgen, R. Molecular analysis and control of cysteine biosynthesis: integration of nitrogen and sulphur metabolism. L. Exp. Bot. 2004, 55, 1283-1292.

(54) Ashida, K.; Iida, S.; Yasui, T. Lack of 26 kDa globulin accompanies increased free amino acid content in rice (Oryza sativa L.) grains. J. Cereal Sci. 2006, 43, 387-392.

(55) Plaxton, W. C. The organization and regulation of plant glycolysis. Annu. Rev. Plant Physiol. Plant Mol. Biol. 1996, 47, 185-214.

(56) Rolletschek, H.; Koch, K.; Wobus, U.; Borisjuk, L. Positional cues for the starch/lipid balance in maize kernels and resource partitioning to the embryo. Plant J. 2005, 42, 69-83.

(57) Mechin, V.; Thevenot, C.; Le Guilloux, M.; Prioul, J.; Damerval, C. Developmental analysis of maize endosperm proteome suggests a pivotal role for pyruvate orthophosphate dikinase. Plant Physiol. 2007, 143, 1203-1219.

(58) Prioul, J.; Méchin, V.; Lessard, P.; Thévenot, C.; Grimmer, M.; Chateau-Joubert, S.; Coates, S.; Hartings, H.; Kloiber-Maitz, M.; Murigneux, A. A joint transcriptomic, proteomic and metabolic analysis of maize endosperm development and starch filling. Plant Biotechnol. J. 2008, 6, 855-869.

(59) Hung, G.-C.; Brown, C. R.; Wolfe, A. B.; Liu, J.; Chiang, H.-L. Degradation of the gluconeogenic enzymes fructose-1,6-bisphosphatase and malate dehydrogenase is mediated by distinct proteolytic pathways and signaling events. L. Biol. Chem. 2004, 279, 49138-49150.

(60) Penfield, S.; Rylott, E. L.; Gilday, A. D.; Graham, S.; Larson, T. R.; Graham, I. A. Reserve mobilization in the arabidopsis endosperm fuels hypocotyl elongation in the dark, is independent of abscisic acid, and requires phosphoenolpyruvate carboxykinase1. $\underline{\text { Plant Cell }}$ 2004, 16, 2705-2718.

PR900954P 